\title{
LETTER TO THE EDITOR Structural insights into the activation of ATM kinase
}

\author{
Cell Research (2019) 29:683-685; https://doi.org/10.1038/s41422-019-0205-0
}

Dear Editor,

ATM (ataxia telangiectasia-mutated) is a master regulator in response to DNA damage and activates downstream pathways involved in cell cycle checkpoints, DNA damage repair, transcription regulation, immune response, central nervous system development and metabolism., ${ }^{1,2}$ Loss of ATM activity in human results in the pleiotropic neurodegeneration disorder ataxia-telangiectasia (A-T) that is characterized by immunodeficiency, cancer predisposition, premature aging and insulin-resistant diabetes. ${ }^{3-5}$ Despite extensive studies over the past two decades, ${ }^{6,7}$ it remains controversial how ATM is activated. Particularly, whether ATM exists in a monomeric form, whether the monomer is more active than dimer, and how dimer-to-monomer transition affects the ATM kinase activity, remain controversial.

To prepare ATM proteins for biochemical and structural studies, we overexpressed human ATM in HEK 293 suspension cells. In most cases, the purified ATM existed in a dimeric form. Monomeric ATM could be obtained when the cells were lysed using homogenizer in a buffer lacking DNase I. The monomeric and dimeric ATM peaks were fairly separated using glycerol gradient centrifugation and the peak fractions were used in the following biochemical and structural analyses (Fig. 1a; Supplementary information, Fig. S1). The generation of monomeric ATM is probably induced by double-strand breaks (DSBs) during cell disruption.

To compare the kinase activities of dimeric and monomeric ATM, we performed an in vitro kinase assay using two representative substrates, the purified human p53 and Chk2 (kinase dead mutant, referred to as Chk2 below). ${ }^{8}$ The monomeric ATM shows kinase activity $\sim 10$-fold higher than the dimeric ATM (Fig. 1b; Supplementary information, Fig. S2a). No obvious difference in the level of S1981 autophosphorylation was observed between the two ATM forms (Supplementary information, Fig. S2b), in agreement with previous studies. ${ }^{9}$ Therefore, ATM monomer is enzymatically more active than dimer and such difference is independent of ATM autophosphorylation at S1981.

We next determined the cryo-EM structures of dimeric and monomeric ATM using single particle reconstructions (Supplementary information, Figs. S3 and S4, Table 1 and movies S1 and S2). The cryo-EM maps of ATM dimer and monomer were refined to 4.3 and $7.8 \AA$ resolution, respectively. The ATM dimer structure reveals a butterfly architecture with two monomers arranged in a two-fold symmetry, indicating a similar fold to that of previously reported ATM dimer in the closed form. ${ }^{10}$ Each monomer has an $\mathrm{N}$-terminal superhelical a-solenoid (designated $\mathrm{N}^{\text {ATM }}$ ), followed by a C-terminal compact core containing the FAT, KD, and FATC domains (designated C ${ }^{\text {ATM }}$ ) (Fig. 1c, d; Supplementary information, Figs. S5 and S6). The FAT domain contains three tetratricopeptide repeat domains (TRD) followed by a short HEAT-repeats domain (HRD) (Fig. 1c, d; Supplementary information, Fig. S6f).

The ATM dimerization is mediated by two $C^{\text {ATM }}$ (Fig. 1d, f). A four-helix bundle (fa19-fa22) of the TRD3 makes extensive contacts with the C-lobe of ATM' (Fig. 1f; Supplementary information, Fig. S6b). The two parallel helices ka10/10' (k represents kinase domain) are sandwiched by helices fa21-22/ fa $21^{\prime}-22^{\prime}$. A loop connecting fa19 and fa 20 packs against the FATC of the KD'. The helices fa21-fa22 of TRD3 and ka9a' and ka10' of the $\mathrm{KD}^{\prime}$ together buttress the kinase domain. The protruded portion of the two long a helices (fa21-fa22) (designated TRD3 dimeric helices, TRD3-DH) packs against two kinase regulatory elements of the other ATM molecule: the $k a 9 b^{\prime}-9 c^{\prime}$ and the activation loop of the KD' (Fig. 1f). The PIKK regulatory domain (PRD) consisting ka9b-9c and the following linker is predicted to prohibit substrate entry and inhibit kinase activity in PIKK kinases. ${ }^{11}$ In ATM dimer, the PRD' is well-ordered because of stabilization by the TRD3-DH and helices ka9a' and ka10' of the $\mathrm{KD}^{\prime}$, supporting an inhibitory function.

The structure of ATM monomer reveals a similar overall fold to one copy of ATM in the dimeric form (Fig. 1e; Supplementary information, Fig. S6d). Compared with ATM dimer, the monomer reveals a more open catalytic pocket due to lack of stabilization by dimer contacts (Fig. 1g, h; Supplementary information, movie S3). In particular, the height of catalytic pocket, as represented by the distance between LBE (LST8 binding element) and HRD, is $53 \AA$ in dimer and $60 \AA$ in monomer. The width of the catalytic pocket, as represented by the distance between ka1 and helices ka10, is $43 \AA$ in dimer and $47 \AA$ in monomer (Fig. 1h). Thus, the associated PRD tends to be more flexible due to less restraint by ka9a and ka10 and lack of stabilization by otherwise associated TRD3-DH' in ATM dimer. The EM density indicates that the PRD is highly dynamic and disordered in ATM monomer, indicating a less restrained catalytic pocket that is more favorable for substrate entry. The conformational switch of PRD is probably the key for the activation of ATM kinase (Fig. 1g-i; Supplementary information, movie S4). Thus, ATM adopts an autoinhibitory conformation in dimeric form and monomerization releases such inhibition and enhances the kinase activity. The pattern of ATM dimerization is generally similar to that of the ATR-ATRIP complex. ${ }^{12}$ However, ATM dimerization is primarily mediated by the upper interface and has less contacts at the lower dimer interface (Supplementary information, Fig. S7a-e). Moreover, ATM monomer has a more open catalytic pocket than the previously reported ATM open dimer (PDB: 5NP1) ${ }^{10}$ (Supplementary information, Fig. S7f-h), suggesting that the open dimer represents a transition state from dimer to monomer.

To test the effect of PRD and TRD3-DH on the dimer-tomonomer transition and on the regulation of kinase activity, we made four internal deletions of ATM: ATM ${ }^{\triangle P R D}$ ( $(2964-2998)$, ATM $^{\triangle P R D-S}(\Delta 2981-2998$, the invisible linker, S represents short), $\mathrm{ATM}^{\triangle \mathrm{DH}}(\Delta 2408-2450, \mathrm{TRD} 3-\mathrm{DH})$, and $\mathrm{ATM}^{\Delta \mathrm{D} H-\mathrm{S}}(\Delta 2422-2435$, the invisible linker) (Fig. 1i). The four mutants were purified using glycerol gradient centrifugation and the fractions at dimer position were used in the in vitro kinase assay. Interestingly, all the four ATM mutants tend to form monomer in solution compared with the wild-type ATM (Supplementary information, Fig. S8), indicating that PRD and TRD3-DH are involved in ATM dimerization. As predicted, ATM ${ }^{\triangle P R D}$ and ATM $^{\triangle P R D-S}$ showed robust activation of kinase activities compared with the wild-type ATM, 


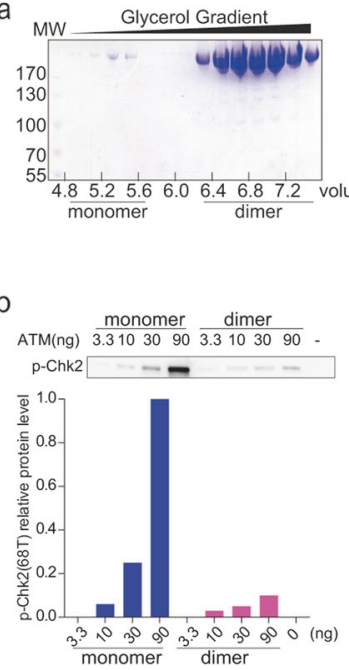

g

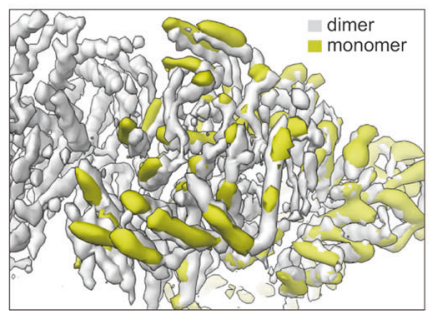

$\mathrm{K}_{\text {dimer(30ng) }++++++++++}$ dsDNA $=\frac{360}{90270540} \frac{950}{90} 270-540 \quad 1800 \quad$ bp

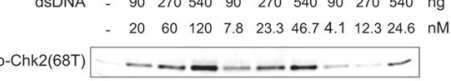

I dimer(30ng) + + + + + + + + + dsDNA $-\frac{360}{90270540} 9 \frac{950}{90270540} \frac{1800}{90270540 \mathrm{ng}} \mathrm{bp}$ $20 \quad 60 \quad 12078 \quad 233467411123240 \quad n$ p-p53(15s)

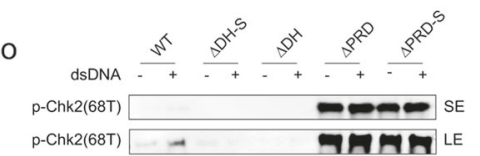

d
C

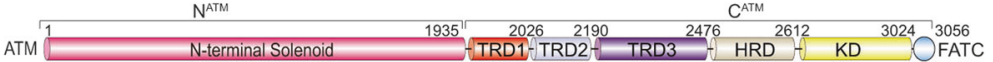

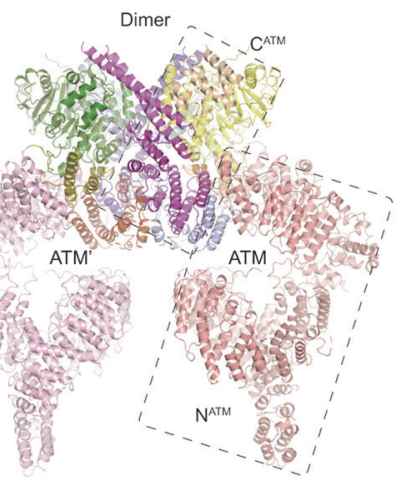

h
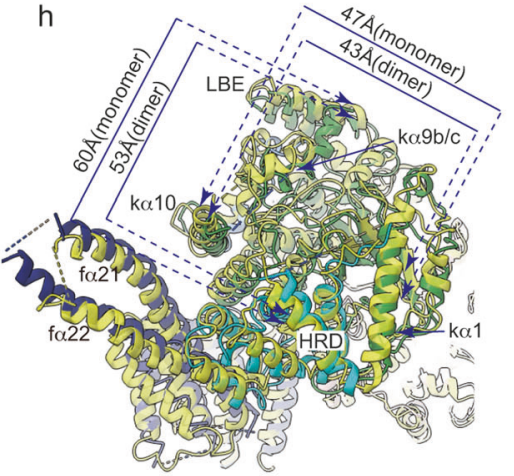

$\mathrm{m}$

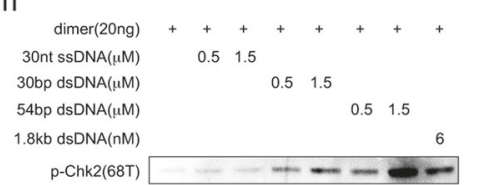

n

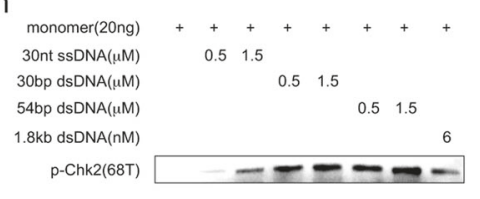

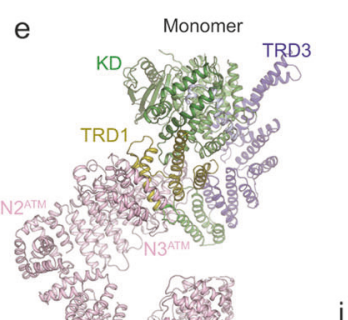
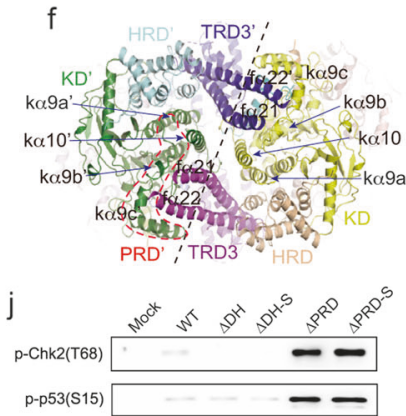

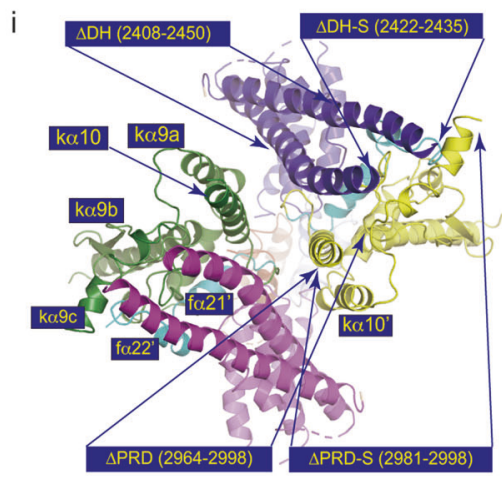

$\mathrm{p}$

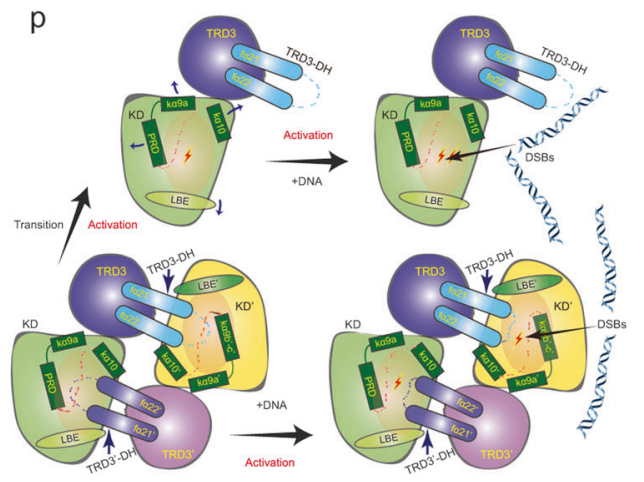

Fig. 1 a Separation of monomeric and dimeric ATM using glycerol gradient centrifugation. The fractions were subjected to SDS-PAGE and stained using Coomassie blue. The fraction volumes were indicated below the figure. $\mathbf{b}$ In vitro kinase assay using increasing amount of purified monomeric and dimeric ATM kinases. The purified human Chk2 $(1 \mu \mathrm{g})$ serves as substrate. The reaction was subjected to SDS-PAGE and visualized by immunoblotting ( $8 \mu \mathrm{L}$ reaction products) with antibody specifically targeting phospho-Thr- 68 of Chk2. Bar representations of the relative kinase activities were generated according to the immunoblotting results. c Color-coded domain structure of human ATM with boundaries indicated with numbers above the schematic representation. The same color scheme is used in all structure figures for one ATM (right one in d) in dimer if not specified elsewhere. The FAT domain consists of TRD1-TRD3 and HRD. We refer to the FAT, KD, and FATC as CATM and the N-terminal solenoid as $\mathrm{N}^{\mathrm{ATM}}$ for simplicity. $\mathbf{d}$-f Ribbon representations of dimeric ATM in front view d, top view $\mathbf{f}$, and ATM monomer e, respectively. In dimeric ATM, the molecule in right side is colored as in c. The monomer ATM is colored in salmon (N ${ }^{\text {ATM }}$ ), olive (TRD1), red (TRD2), blue (TRD3), cyan (HRD), green (KD), and lightblue (FATC), respectively. g, h Structural comparison of ATM dimer and monomer. CryoEM maps $\mathbf{g}$ and ribbon representations $\mathbf{h}$ of ATM dimer and monomer are shown in a similar view. One ATM molecule in the dimer is omitted in $\mathbf{g}$ for clarity. The ATM dimer is colored in yellow. The heights (distance between C $\alpha$ atoms of residue D2597 and D2795) and widths (distance between $\mathrm{C} \alpha$ atoms of residue M3011 and A2626) of the catalytic pockets of ATM dimer and monomer are indicated for comparison. $\mathbf{i}$ A top view structure of ATM dimer showing critical regulatory elements for dimerization and kinase activity. The regions deleted in the four ATM mutants are indicted with arrows. $\mathbf{j}$ In vitro kinase assay using purified wild-type ATM and four ATM mutants in equal concentration (20 $\mathrm{ng}$ ). The assays were performed as in $\mathbf{b}$. The purified human Chk2 $(1 \mu \mathrm{g})$ and $\mathrm{p} 53(0.7 \mu \mathrm{g})$ were used as substrates, respectively. The activities were visualized with antibodies against phospho-Thr-68 of Chk2 and phospho-Ser-15 of p53, respectively. $\mathbf{k}$, I The in vitro kinase assays were performed using dimeric ATM $(30 \mathrm{ng})$ in the presence of increasing amount of DNA fragments in three different lengths. The lengths and amounts of DNA were indicated, and $1 \mu \mathrm{g}$ purified human Chk2 $\mathbf{k}$ and $0.7 \mu \mathrm{g} \mathrm{p} 53 \mathrm{I}$ serves as substrates in $20 \mu \mathrm{L} 50 \mathrm{mM} \mathrm{KCl}$ system. The reactions were subjected to SDS-PAGE for immunoblotting with the activities detected by antibodies against phospho-Thr-68 of Chk2 $\mathbf{k}$ and phospho-Ser-15 of p53 I. $\mathbf{m}, \mathbf{n}$ Short DNA could activate ATM dimer $\mathbf{m}$ and monomer $\mathbf{n}$. The kinase assays were performed as in $\mathbf{k}$ with the lengths and amounts of DNA indicated. $o$ The in vitro kinase assays were performed using equal amount (20 ng) of purified wild-type ATM and ATM mutants in the absence or presence of $1.8 \mathrm{~kb}$ dsDNA (500 ng). SE short exposure, LE long exposure. p A working model that illustrates the mechanism for ATM activation in response to DNA damage (see the main text for details) 
supporting the key role of PRD in inhibiting the kinase activity (Fig. 1j). Unexpectedly, ATM $^{\Delta D H}$ and $A T^{\Delta D H-S}$ showed similar (p53 as substrate) or even decreased (Chk2 as substrate) kinase activities compared with the wild-type ATM (Fig. 1j). The deletion of TRD3-DH may not be sufficient to release the inhibition by PRD, which is still supported by helices ka9a and ka10. Alternatively, the TRD3-DH might also be required for phosphorylation of substrates like Chk2.

Biochemical studies have shown that ATM can be activated not only by MRN complex, but also by individual DNA (Supplementary information, Fig. S9). The ATM was activated by dsDNA in a manner dependent on the concentration and type of free DNA ends (Fig. 1k-m; Supplementary information, Fig. S10). Although ATM monomer is enzymatically more active than ATM dimer (Fig. 1b), ATM monomer activity can be further stimulated by DNA fragments (Fig. 1n), indicating that DNA stimulates ATM activity independently from oligomerization state. The four ATM mutants, $\mathrm{ATM}^{\triangle \mathrm{PRD}}, \mathrm{ATM}^{\triangle \mathrm{PRD}-\mathrm{S}}, \mathrm{ATM}^{\triangle \mathrm{DH}}$ and $\mathrm{ATM}^{\triangle \mathrm{DH}-\mathrm{S}}$ could not be stimulated by dsDNA' (Fig. 10), suggesting that these regulatory elements (PRD and TRD3-DH) are required for DNA-mediated activation. Consistently, oxidative crosslinking of C2291/C2291' may lead to close association of two PRD domains and autoinhibition release in the context of ATM dimer. ${ }^{13}$ Therefore, the above observations indicate a combinatorial mechanism for activation of ATM, the dimer-to-monomer transition and DNAmediated activation.

We here proposed a model for ATM activation in response to DNA damage (Fig. 1p). The ATM dimer adopts an autoinhibitory fold, in which the PRD is restrained in an inhibitory conformation by ka9a, ka10 and TRD3-DH'. The well-positioned PRD prohibits substrate entry and inhibits kinase activity of ATM. In monomeric ATM, the PRD is more flexible due to lack of stabilization by TRD3$\mathrm{DH}$ and less support by ka9a and ka10. The ATM monomer can be further activated by dsDNA. These regulatory elements (PRD and TRD3-DH) are also required for DNA-mediated ATM activation, suggesting that DNA may also regulate the conformation of PRD and TRD3-DH for activation. Thus, dimer-to-monomer transition and DNA association independently release the inhibitory conformation of PRD and collectively lead to a fully activated ATM. Upon DNA damage, MRN and other factors may facilitate the recruitment of ATM to the broken DNA ends, which directly activates ATM kinase activity for substrate phosphorylation.

The cryo-EM maps have been deposited in the EM Databank under accession numbers: EMD-9950 (human ATM dimer), EMDB9951 (core region of ATM dimer) and EMDB-9949 (human ATM monomer). The coordinates of structural models have been deposited in the Protein Data Bank under accession numbers: 6K9L (human ATM dimer) and 6K9K (human ATM monomer).

\section{ACKNOWLEDGEMENTS}

We thank Center for Biological Imaging (CBI) of Institute of Biophysics (IBP) of Chinese Academy of Sciences (CAS) and National Center for Protein Science Shanghai (NCPSS) for the support on cryo-EM data collection and data analyses. We thank staff members working in Biomedical Core Facility, Fudan University for their help on Mass Spectrometry analyses. This work was supported by grants from the Ministry of Science and Technology of China (2016YFA0500700), the National Natural Science Foundation of China (31830107, 31821002, 31425008), the National Program for support of Top-Notch Young Professionals (Y.X.), the Strategic Priority Research
Program of the Chinese Academy of Sciences (grant no. XDB08000000), and the UK Wellcome Trust Investigator Award 206422/Z/17/Z (P.Z.). We acknowledge Diamond for access of the Cryo-EM facilities at the UK national electron bio-imaging centre (eBIC, proposal NT21004), funded by the Wellcome Trust, MRC and BBSRC.

\section{AUTHOR CONTRIBUTIONS}

J.X., M.L., Y.Q., and Y.X. designed the experiments. J.X., C.G., and Y.Q. purified the proteins. M.L., Y.C., Y.Q., P.Z., J. X., Y.T., and Z.Y. prepared the cryo-EM sample, collected the data and determined the structures. M.L., J.L., J. X. built the structural model. J. X., B. P., and Y. X. performed biochemical analyses. J. X., and Y.X. analyzed the data and wrote the manuscript with support from all the authors. Y.X. supervised the project.

\section{ADDITIONAL INFORMATION}

Supplementary information accompanies this paper at https://doi.org/10.1038/ s41422-019-0205-0.

Competing interests: The authors declare no competing interests.

Jianxiong Xiao ${ }^{1,2}$, Mengjie Liu ${ }^{1,2}$, Yilun Qi ${ }^{1}$, Yuriy Chaban ${ }^{3}$, Chao Gao', Beiqing Pan ${ }^{1}$, Yuan Tian ${ }^{1}$, Zishuo $\mathrm{Yu}^{1}$, Jie $\mathrm{Li}^{4}$ Peijun Zhang ${ }^{3,5}$ and Yanhui Xu (iD ${ }^{1,2,6,7}$

${ }^{1}$ Fudan University Shanghai Cancer Center, Institutes of Biomedical Sciences, State Key Laboratory of Genetic Engineering and Key Laboratory of Medical Epigenetics and Metabolism, Shanghai Medical College of Fudan University, Shanghai 200032, China; ${ }^{2}$ Key Laboratory of Molecular Medicine, Ministry of Education, Department of Systems Biology for Medicine, School of Basic Medical Sciences,

Shanghai Medical College of Fudan University, Shanghai 200032, China; ${ }^{3}$ Electron Bio-Imaging Centre, Diamond Light Sources, Harwell Science and Innovation Campus, Didcot, Oxfordshire, UK; ${ }^{4}$ National Facility for Protein Science in Shanghai, Zhangjiang Lab, Shanghai 201210, China; ${ }^{5}$ Division of Structural Biology, Wellcome Trust Centre for Human Genetics, University of Oxford, Oxford, UK; ${ }^{6}$ Collaborative Innovation Center of Genetics and Development, School of Life Sciences, Fudan University, Shanghai 200433, China and ${ }^{7}$ CAS Center for Excellence in Molecular Cell Science, Chinese Academy of Sciences, Shanghai 200031, China

These authors contributed equally: Jianxiong Xiao, Mengjie Liu, Yilun Qi, Yuriy Chaban, Chao Gao. Correspondence: Yanhui Xu (xuyh@fudan.edu.cn)

\section{REFERENCES}

1. Bhatti, S. et al. Cell Mol. Life Sci. 68, 2977-3006 (2011).

2. Jackson, S. P. \& Bartek, J. Nature 461, 1071-1078 (2009).

3. Lavin, M. F. Nat. Rev. Mol. Cell Biol. 9, 759-769 (2008).

4. McKinnon, P. J. Annu. Rev. Pathol. 7, 303-321 (2012).

5. Wang, X. et al. Nat. Commun. 7, 11655 (2016).

6. Adamowicz, M. J. Immunol. Sci. 2, 26-31 (2018).

7. Paull, T. T. Annu. Rev. Biochem 84, 711-738 (2015).

8. Brown, A. L. et al. Proc. Natl Acad. Sci. USA 96, 3745-3750 (1999).

9. Dupre, A., Boyer-Chatenet, L. \& Gautier, J. Nat. Struct. Mol. Biol. 13, 451-457 (2006).

10. Baretic, D. et al. Sci. Adv. 3, e1700933 (2017).

11. Yang, H. et al. Nature 497, 217-223 (2013).

12. Rao, Q. et al. Cell Res. 28, 143-156 (2017).

13. Guo, Z., Kozlov, S., Lavin, M. F., Person, M. D. \& Paull, T. T. Science 330, 517-521 (2010). 